\title{
Applications of a non-interferometric $x$-ray phase contrast imaging method with both synchrotron and conventional sources
}

\author{
M. Endrizzi, P. C. Diemoz, P. R. T. Munro, C. K. Hagen, M. B. Szafraniec, T. P. \\ Millard, C. E. Zapata, R. D. Speller and A. Olivo \\ Department of Medical Physics and Bioengineering, University College London \\ Malet Place, Gower Street, London WC1E 6BT, UK \\ *E-mail: a.olivo@ucl.ac.uk
}

\begin{abstract}
We have developed a totally incoherent, non-interferometric $\mathrm{x}$-ray phase contrast imaging (XPCI) method. This is based on the edge illumination (EI) concept developed at the ELETTRA synchrotron in Italy in the late ' $90 \mathrm{~s}$. The method was subsequently adapted to the divergent beam generated by a conventional source, by replicating it for every detector line through suitable masks. The method was modelled both with the simplified ray-tracing and with the more rigorous wave-optics approach, and in both cases excellent agreement with the experimental results was found. The wave-optics model enabled assessing the methods' coherence requirements, showing that they are at least an order of magnitude more relaxed than in other methods, without this having negative consequences on the phase sensitivity. Our masks have large pitches (up to 50 times larger than in grating interferometry, for example), which allows for manufacturing through standard lithography, scalability, cost-effectiveness and easiness to align. When applied to a polychromatic and divergent beam generated by a conventional source, the method enables the detection of strong phase effects also with uncollimated, unapertured sources with focal spots of up to $100 \mu \mathrm{m}$, compatible with the stateof-the-art in mammography. When used at synchrotrons, it enables a contrast increase of orders of magnitude over other methods. Robust phase retrieval was proven for both coherent and incoherent sources, and additional advantages are compatibility with high x-ray energies and easy implementation of phase sensitivity in two directions simultaneously. This paper briefly summarizes these achievements and reviews some of the key results.
\end{abstract}

KEYWORDS: X-ray imaging; Phase-contrast imaging; X-ray masks.

\footnotetext{
${ }^{*}$ Corresponding author.
} 


\section{Contents}

$\begin{array}{lr}\text { 1. Introduction } & 1\end{array}$

2. Materials and Methods $r$

3. Results $\quad 4$

4. Conclusions $\quad 6$

\section{Introduction}

X-ray phase contrast imaging (XPCI) exploits interference and refraction effects ("phase" effects, hence the name) instead of attenuation to generate image contrast. By doing this, it both enhances the visibility of all detail in an x-ray image, and it enables the visualization of features classically considered $\mathrm{x}$-ray invisible due to lack of attenuation contrast. The rationale behind this is that, considering the real and imaginary parts of the refractive index $n$ [1]:

$$
n=1-\delta+i \beta
$$

it can be noted that the unit decrement of the real part $\delta$ (responsible for phase effects) is much larger than the imaginary part $\beta$ (responsible for attenuation), typically up to 1000 times larger for most materials and over a wide range of $\mathrm{x}$-ray energies.

Initially this was exploited either through free space propagation $[2,3]$ or perfect crystals ("analyser based" imaging, [4-6]). This works very well with synchrotron x-ray beams, which typically possess both spatial and temporal coherence. Outside synchrotrons, effective implementations of XPCI become more difficult. Free-space propagation methods tolerate relaxed temporal coherence conditions [3], but suffer strongly from reductions in spatial coherence: as the source dimensions are made larger, phase effects rapidly vanish [7-11]. This means that microfocal sources can be used as an alternative to synchrotrons, but at the price of long exposure times (hours, [3]), due to their low emitted flux. Crystal methods, on the other hand, can tolerate a relaxed degree of spatial coherence [12], but intrinsically impose high temporal coherence, as the crystal automatically selects a narrow bandwidth out of the spectrum emitted by the source.

In the late nineties, we were trying to solve this conundrum at the SYRMEP (Synchrotron Radiation for MEdical Physics) beamline of the ELETTRA synchrotron in Trieste, Italy, by combining the aspects of free-space propagation that make it resilient to reduced temporal coherence with those that enable crystal methods to tolerate somewhat relaxed spatial coherence. The latter aspect is due to the fact that crystals can be seen as very effective angular filters, and can thus be used to exploit refraction effects with high accuracy. However, having to employ a crystal practically forces the use of monochromatic radiation. The goal was therefore 
to develop an approach that could perform a fine angular selection on refracted $\mathrm{x}$-rays without employing a crystal. This was achieved by means of the edge illumination (EI) method, which consists in illuminating only the edges of the detector pixels [13]. In this way, small deviations in the x-ray direction are sufficient to deflect x-rays out of the detector active surface causing dark fringes, and vice-versa (see fig. 1 below). Images with a strong similarity to those provided by analyser based imaging are obtained in this way [13], but the absence of the crystal makes the method amenable to divergent and polychromatic beams. This is achieved by means of two aperture masks [14-15], placed one before and one after the imaged sample (sometimes referred to as "coded apertures", although they differ significantly from those used in nuclear medicine or astronomy), which enable repeating the EI configuration for every row (or column) of pixel in an area detector (see fig. 2 below).

It should be noted that, despite some superficial similarity in the setup with grating (or "shearing", or "Talbot") interferometry [16-18], the method is based on a different physical principle (EI instead of Talbot self-imaging), and is in fact a totally incoherent method, while grating interferometry still requires at least spatial coherence, which (in the Talbot/Lau configuration [19]) can also be achieved through the introduction of the "source" grating [20].

While in grating interferometry the gratings have pitches of the order of a few micron, thus requiring highly specialized fabrication facilities and very fine alignments (of down to a few tens of $\mathrm{nm}$ [21]), our masks have pitches up to 50 times larger, which allows for manufacturing through standard lithography, scalability, cost-effectiveness and easiness to align. The larger pitch, together with the fact that stepping one mask with respect to the other is not required, relaxes the alignment requirements to a couple of $\mu \mathrm{m}$ [22]. Moreover, the presample mask (see figure 2) protects the sample from unwanted radiation, enabling the achievement of clinically acceptable doses [23].

Most importantly, this set-up provides strong phase contrast signals with focal spots of up to $100 \mu \mathrm{m}$ [15], compatible with current state-of-the-art mammography sources, without requiring excessive source-to-sample distances. Together with the fact that the full, unfiltered polychromatic beam produced by a Mo $[14-15,23]$ or a $\mathrm{W}[22,24]$ source can be used, this means that ours is the first XPCI method working with sources that are simultaneously incoherent both spatially and temporally. Despite this, phase sensitivity at least equivalent to that of grating interferometers operated on the $3^{\text {rd }}$ Talbot order was recently demonstrated [23].

\section{Materials and Methods}

In its basic, synchrotron implementation the EI method is extremely simple (see figure 1).

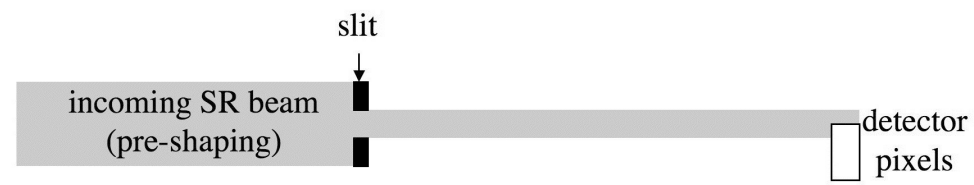

Figure 1. The basic implementation of the edge illumination method with synchrotron radiation.

All it requires is beam pre-shaping through a slit (the vertical aperture of which determines the phase sensitivity together with the sample to detector distance $[13,15])$, and a "sensitivity 
edge" on the detector surface. If a "linear" detector consisting of a single row of pixels is available, the edge of the pixel row itself can be used (like in figure 1). With a more conventional detector, a polished absorbing edge (e.g. made of tungsten carbide) can be mounted in front of the detector and aligned with a row of pixels, to create a sharp transition between sensitive and insensitive detector areas [25]. The beam collimated by the "shaping" slit is then positioned such that it straddles the edge between sensitive and insensitive regions on the detector. In this synchrotron setup, where the beam is often very thin in the vertical direction, samples are typically scanned through the beam to obtain the two-dimensional images. In our case, the sample is scanned immediately downstream of the "shaping" slit. During this scan, when one of the details in the sample touches the upper part of the beam, it can refract x-rays downwards (with respect to figure 1). These x-rays, which would normally miss the pixel, are thus counted. The number of counts corresponding to that sample position would therefore be increased, creating a bright fringe along that side of the detail. Likewise, when the detail grazes the bottom part of the beam, x-rays that are normally counted can be deviated upwards and thus possibly miss the detector, creating a negative fringe. "Differential" (i.e. proportional to the first derivative of the phase shift) XPCI images are obtained in this way, just like the ones obtained on one side of the reflectivity curve of an analyser crystal [26]. Although in our case the differential nature is due to the convolution of "half" a free-space propagation profile with the pixel response function [27-28], it can be shown that, by optimizing beam thickness and sample-to-detector distance so that comparable phase sensitivity is achieved, results effectively equivalent to analyser based imaging are obtained [13].

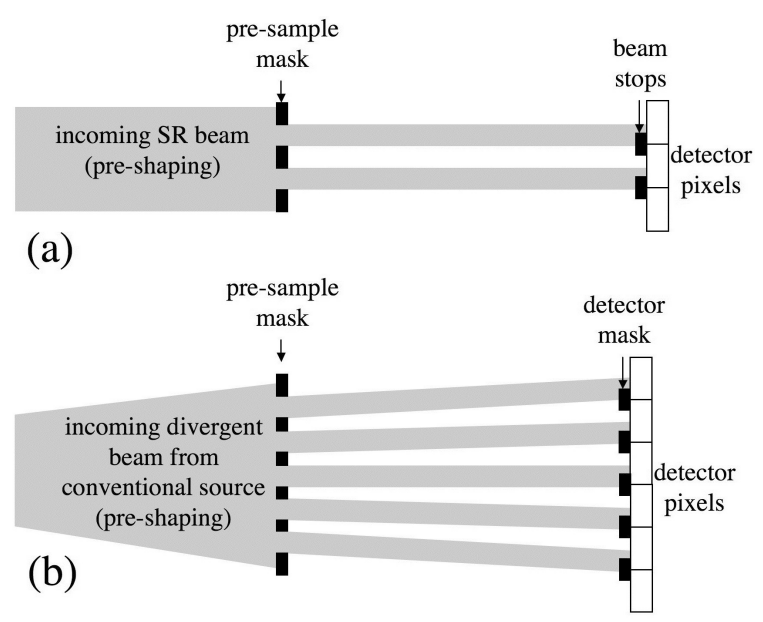

Figure 2. Extension of the edge illumination method to larger beam cross-sections: (a) and (b) show the parallel (typically synchrotron) and divergent (typically conventional source) beam configurations, respectively.

The edge illumination concept is easily extended to larger beam cross-sections by means of masks. Figure 2 shows possible embodiments for a parallel beam (figure 2a), e.g. a vertically larger synchrotron beam, and for a divergent beam originated from a conventional source (figure $2 b$ ). The latter simply requires rescaling the pre-sample mask to account for the beam divergence. In both cases, the sample is placed immediately downstream of the pre-sample mask. An additional mask (or series of beam-stops) is placed in contact with the detector to create insensitive regions between adjacent pixels, which allow realizing the EI condition. The availability of a plurality of beams allows avoiding sample scanning; however, "dithering" (i.e. 
interweaving more images acquired while displacing the sample by sub-pixel quantities) enables overcoming the resolution limit imposed by the pixel size $[15,22]$. The fact that the detector mask redefines the response function of the detector pixel (effectively shrinking its point spread function) means that the application of deconvolution methods is not required, provided the dithering step is larger than or equal to the apertures in the detector mask.

While the system represented in figure 1 is used at various synchrotrons including ELETTRA [29], the ESRF [25] and Diamond (see Diemoz et al. paper in this volume), using either sets of Huber slits or the beamline air slits as pre-sample beam shapers and polished tungsten edges as beam stops, two systems based on conventional sources (figure 2b) are currently in operation in our labs at UCL. One is based on a Rigaku M007 Mo source, with a focal spot of approximately $70 \mu \mathrm{m}$, typically operated at $25 \mathrm{~mA}$ and 35 or $40 \mathrm{kVp}$. This is used primarily for medical and biological applications, and it uses the ANRAD "SMAM" a-Se flat panel detector, which has a pixel size of $85 \mu \mathrm{m}$. The other system is based on an X-Tek W source with a focal spot of $50 \mu \mathrm{m}$, operated between 40 and $100 \mathrm{keV}$ and at $1 \mathrm{~mA}$, and it features the Hamamatsu C9732DK CMOS-based flat panel detector with a $50 \mu \mathrm{m}$ pixel. This is typically used for industrial and material science applications. Different sets of masks are available for both systems, with apertures shaped like long slits matching detector columns (or rows), or like "L's" matching each detector pixel, for simultaneous two-directional phase sensitivity [30]. In all cases, gold layers between 30 and $200 \mu \mathrm{m}$ in thickness are electroplated on thin, low absorbing graphite substrates. All masks were manufactured to the authors' design by Creatv Microtech (Potomac, MD). While all detector masks for the Mo system have a pitch matching the ANRAD pixel size $(85 \mu \mathrm{m})$, masks with pitches of both $50 \mu \mathrm{m}$ ("non-skipping" masks) and $100 \mu \mathrm{m}$ ("skipping" masks) are available for the $\mathrm{W}$ system, corresponding to once and twice the pixel pitch of the Hamamatsu detector, respectively. Being an indirect detection system, the Hamamatsu detector features significant signal spillover between adjacent pixels. With our method, this results in a contrast reduction for non-dithered images, and can result in artefacts for dithered images [31]. The problem is solved by using a detector mask with twice the pixel pitch, as the spillover into the second neighbour is negligible. This has a price to pay in terms of resolution, which can be overcome by dithering; however, the acquisition time increases with the dithering steps. Hence, non-skipping masks are used when resolution and shorter acquisition times are more important than contrast maximization. This problem does not apply to the Mo system, as the signal spillover is almost negligible for the ANRAD which is a direct conversion detector; hence, only non-skipping masks are used. All masks are mounted on stacks of Newport translators and Kohzu cradles for alignment. Samples are mounted on an additional translation stage, which is used either to perform sample "dithering" or to move the sample out of the field of view when flat field images are acquired. The sample stage on the Mo system also features a rotary stage to perform CT acquisitions; this is mounted on two cradles that are used to align the axis of rotation with the detector columns. An upgrade is currently underway to have the same feature available also in the $\mathrm{W}$ system.

\section{Results}

The method was modelled both with the simplified ray-tracing $[13,15]$ and with the more rigorous wave-optics approach [32], and in both cases excellent agreement with the experimental results was found. The wave-optics model enabled assessing the method's 
coherence requirements, showing that they are at least one order of magnitude more relaxed than in other approaches [33], without this having negative consequences on the phase sensitivity [23, 34]. A robust phase-retrieval method was developed, based on the processing of two images acquired while illuminating opposite sides of the apertures on the detector mask (see figure 3).

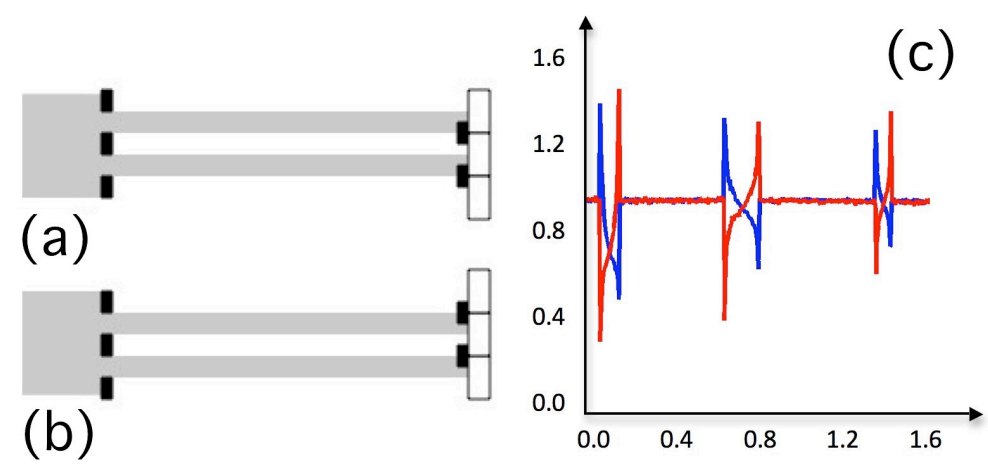

Figure 3. Phase retrieval in edge illumination $x$-ray phase contrast imaging. (a) and (b) show the two mask configurations required to acquire two images that can be processed to extract phase and absorption. (c) shows profiles extracted from the images of three thin wires (from left to right, $100 \mu \mathrm{m} \mathrm{Al}, 200 \mu \mathrm{m}$ PEEK and $100 \mu \mathrm{m}$ PEEK) imaged in the two configurations. Relative intensity is plotted as a function of spatial displacement in $\mathrm{mm}$.

The two symmetric mask configurations result in inverting the differential phase signal while leaving absorption unchanged, again in perfect analogy with analyser based imaging: by looking at the profiles in figure $3 \mathrm{c}$, one can immediately spot the similarity with images of the same samples acquired on the two sides of the crystal reflectivity curve in analyser based imaging [26]. However, by applying appropriate corrections for the extended source size, in our case the method was shown to provide reliable quantitative results also with spatially and temporally incoherent sources [34]. Figure 4 shows an example of "incoherent" phase and absorption separation obtained with the UCL Mo-based system.

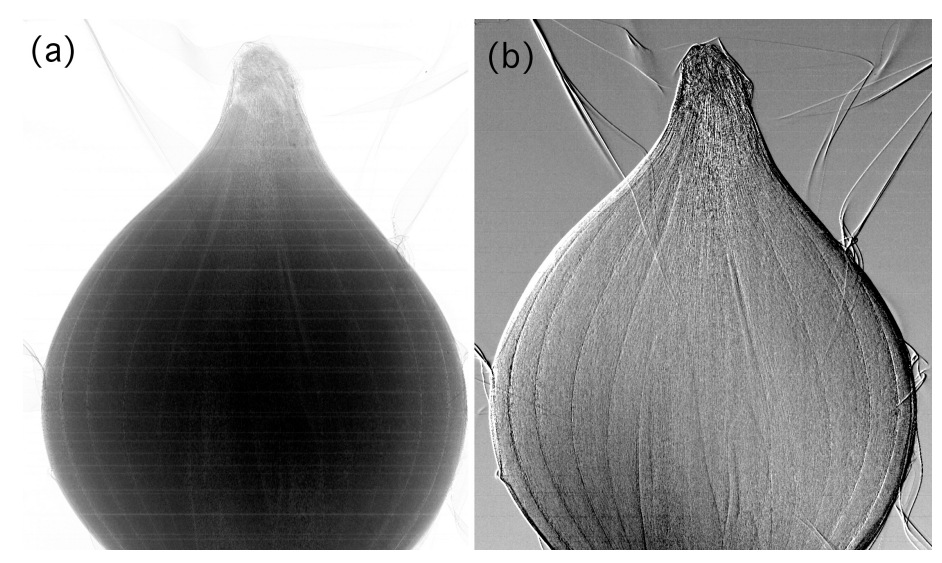

Figure 4. Retrieved absorption (a) and differential phase (b) images of a small onion $(\sim 2 \mathrm{~cm})$, obtained with a temporally and spatially incoherent source operated at $35 \mathrm{kVp}$ and $25 \mathrm{~mA}$. 
This retrieval method was extended to include a correction for the gradient of absorption, which enables precise phase retrieval also for strongly absorbing objects. This algorithm refinement also enables reliable phase retrieval along the edges of details, where the very high gradients in the $\delta$ values cause other retrieval algorithms to break down [29, 34]. Effectively, in reference [34] we carry out a direct comparison between phase profiles of the same samples retrieved at synchrotrons and with conventional sources, showing that, at least for the simple objects examined in that case, comparable results are obtained.

The method was used to image a variety of samples including breast tissue, murine cartilage, explosives, fossils, small animals, defects and blemishes in composite materials - demonstrating in all cases high contrast increases over conventional x-ray methods. Following the development of the phase retrieval algorithm, which gives access to quantitative information, the method was extended to CT; one example is shown in figure 5.
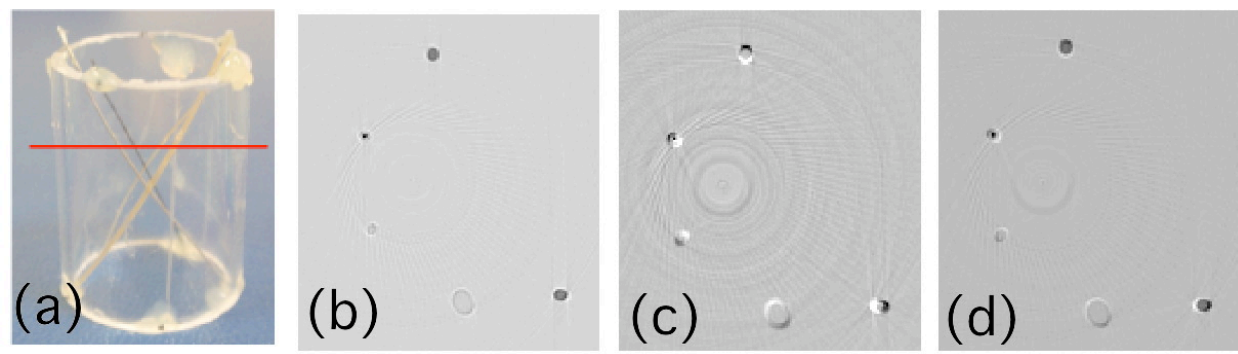

Figure 5. CT implementation of the edge-illumination method. (a) photograph of the used wire sample, (b) reconstruction of the absorption signal, (c) reconstruction of the phase gradient signal, (d) reconstruction of the "mixed" signal (see text).

Alongside the reconstruction of phase and absorption signals, figure 5 also shows the option to reconstruct a "mixed" CT volume (figure 5d), where every voxel contains a weighted average of phase and absorption, as recently demonstrated by Diemoz et al [35]. This enables reconstructing a reliable $3 \mathrm{D}$ volume, with enhanced detail visibility, with a single CT acquisition over $180^{\circ}$. As recently discussed by Zhu et al [36], rotation over $360^{\circ}$ would again enable separating phase and absorption, as the two "symmetric" $180^{\circ}$ series of projections effectively correspond to two separate $180^{\circ}$ acquisitions with the pre-sample mask in the two positions shown in figures $3 \mathrm{a}$ and $3 \mathrm{~b}$.

Finally, when used with coherent sources (eg synchrotrons), the method's flexibility and capacity to "amplify" the phase contrast by reducing the illuminated fraction of the pixels allows significant contrast increases over other XPCI methods. This was recently demonstrated at the ESRF, where we obtained contrast values between 20 and 30 times higher than free-space propagation XPCI at very high x-ray energy $(85 \mathrm{keV},[25])$. This concept was then translated to other synchrotrons setups and different energy ranges, constantly demonstrating substantially increased sensitivity; this topic is discussed in detail in the contribution by Diemoz et al in this same volume.

\section{Conclusions}

This paper briefly reviews some of our recent achievements with the edge illumination XPCI method. When implemented with conventional x-ray sources, the method provides intense 
phase contrast signal and quantitative phase retrieval with focal spots of up to at least $100 \mu \mathrm{m}$ (without this imposing large source-to-sample distances). With coherent sources (e.g. synchrotrons), it enables contrast (and thus sensitivity) amplification over other XPCI approaches. The method is simple, robust, effective at very high x-ray energies [24-25], and resilient to environmental vibrations. The implementation with conventional sources is based on the realization of x-ray masks which are cheap, easy to fabricate and scalable to large sizes. Their graphite substrates are practically x-ray transparent, and only one such substrate is placed downstream of the sample. The pre-sample mask can be used to prevent to a good extent the delivery of unwanted dose (for example by using small lateral displacements between presample and detector mask), and the use of relatively large apertures in thin gold layers means that almost no angular filtration is applied to the divergent beam (e.g., in our case the beam intensity does not "drop" at the sides of the field of view because of higher mask absorption for photons emitted at increasing angles with respect to the optical axis). This results in a highly efficient use of the flux generated by the source, potentially making this the technology of choice for clinical/industrial translations of XPCI. While so far we have been focusing mostly on the technical development of the method, with only limited biomedical applications demonstrated so far [23], the next stages of our research will be dedicated directly to the validation of the method on a range of significant applications in medicine and biology. On the other hand, we will also be further developing synchrotron implementations, as the increased sensitivity could be used to explore new scientific applications that are currently inaccessible.

\section{Acknowledgments}

This work is funded by the UK Engineering and Physical Sciences Research Council (Grants $\mathrm{EP} / \mathrm{G} 004250 / 1$ and EP/I021884/1).

\section{References}

[1] M. Born and E. Wolf, Principles of optics, $6^{\text {th }}$ ed, Pergamon, Oxford, 1980.

[2] A. Snigirev et al., On the possibilities of $x$-ray phase contrast microimaging by coherent highenergy synchrotron radiation, Rev. Sci. Instrum. 66 (1995) 5486.

[3] S. W. Wilkins et al., Phase-contrast imaging using polychromatic hard x-rays, Nature 384 (1996) 335.

[4] E. Forster, K. Goetz and P. Zamumseil, Double crystal diffractometry for the characterization of targets for laser fusion experiments, Krist. Tech. 15 (1980) 937.

[5] T. J. Davis et al., Phase-contrast imaging of weakly absorbing materials using hard x-rays, Nature 373 (1995) 595.

[6] V. N Ingal and E. A. Beliaevskaya, X-ray plane-wave topography observation of the phase contrast from a non-crystalline object, J. Phys. D: Appl. Phys. 28 (1995) 2314.

[7] F. Arfelli et al., Low-dose phase-contrast x-ray medical imaging, Phys. Med. Biol. 43 (1998) 2845.

[8] A. Olivo, Towards the exploitation of phase effects in clinical synchrotron radiation radiology, Nucl. Instrum. Meth. A 548 (2005) 194. 
[9] A. Olivo and R. Speller, Experimental validation of a simple model capable of predicting the phase contrast imaging capabilities of an x-ray imaging system, Phys. Med. Biol. 51 (2006) 3015.

[10] T. Gureyev et al., Some simple rules for contrast, signal-to-noise and resolution in in-line x-ray phase contrast imaging, Opt. Exp. 16 (2008) 3223.

[11] T. Gureyev et al., Refracting Röntgen's rays: propagation-based x-ray phase contrast for biomedical imaging, J. Appl. Phys. 105 (2009) 102005.

[12] D. Vine et al., Analyzer-based phase contrast imaging and phase retrieval using a rotating anode $x$ ray source, Appl. Phys. Lett. 91 (2007) 254110.

[13] A. Olivo et al., An innovative digital imaging set-up allowing a low-dose approach to phase contrast applications in the medical field, Med. Phys. 28 (2001) 1610.

[14] A. Olivo and R. Speller, A coded-aperture approach allowing $x$-ray phase contrast imaging with conventional sources, Appl. Phys. Lett. 91 (2007) 074106.

[15] A. Olivo and R. Speller, Modelling of a novel x-ray phase contrast imaging technique based on coded apertures, Phys. Med. Biol. 52 (2007) 6555.

[16] C. David, B. Nohammer, H. H. Solak and E. Ziegler, Differential x-ray phase contrast imaging using a shearing interferometer, Appl. Phys. Lett. 81 (2002) 3287.

[17] A. Momose et al., Demonstration of x-ray Talbot interferometry, Jpn. J. Appl. Phys. 42 (2003) L866.

[18] T. Weitkamp et al., X-ray phase imaging with a grating interferometer, Opt. Exp. 13 (2005) 6296.

[19] W. B. Case, M. Tomandl, S. Dechapunya and M. Arndt, Realization of optical carpets in the Talbot and Talbot-Lau configurations, Opt. Exp. 17 (2009) 20966.

[20] F. Pfeiffer, T. Weitkamp, O. Bunk and C. David, Phase retrieval and differential phase-contrast imaging with low-brilliance x-ray sources, Nat. Phys. 2 (2006) 258.

[21] J. Zambelli, N. Bevins, Z. Qi and G. H. Chen, Radiation dose efficiency comparison between differential phase contrast CT and conventional absorption CT, Med. Phys. 37 (2010) 2473.

[22] A. Olivo, K. Ignatyev, P. R. T. Munro and R. Speller, Noninterferometric phase-contrast images obtained with incoherent x-ray sources, Appl. Opt. 50 (2011) 1765.

[23] M. Marenzana et al., Visualization of small lesions in rat cartilage by means of laboratory-based $x$ ray phase contrast imaging, Phys. Med. Biol. 57 (2012) 8173.

[24] K. Ignatyev et al., Coded apertures allow high-energy x-ray phase contrast imaging with laboratory sources, J. Appl. Phys. 110 (2011) 014906.

[25] A. Olivo, P. C. Diemoz and A. Bravin, Amplification of the phase contrast signal at very high x-ray energies, Opt. Lett. 37 (2012) 915.

[26] D. Chapman et al., Diffraction enhanced x-ray imaging, Phys. Med. Biol. 42 (1997) 2015.

[27] A. Olivo and R. Speller, Image formation principles in coded-aperture based x-ray phase contrast imaging, Phys. Med. Biol. 53 (2008) 6461. 
[28] P. R. T. Munro et al., "Edge illumination" in x-ray phase contrast imaging, AIP Conf. Proc. 1446 (2012) 118.

[29] P. R. T. Munro et al., A quantitative, non-interferometric $x$-ray phase contrast imaging technique, Opt. Exp., in press.

[30] A. Olivo et al., A non-free-space propagation x-ray phase contrast imaging method sensitive to phase effects in two directions simultaneously, Appl. Phys. Lett. 94 (2009) 044108.

[31] K. Ignatyev, P. R. T. Munro, R. D. Speller and A. Olivo Effects of signal diffusion on x-ray phase contrast images, Rev. Sci. Instrum. 82 (2011) 073702.

[32] P. R. T. Munro, K. Ignatyev, R. D. Speller and A. Olivo, The relationship between wave and geometrical optics models of code aperture type x-ray phase contrast imaging systems, Opt. Exp. 18 (2010) 4103.

[33] P. R. T. Munro, K. Ignatyev, R. D. Speller and A. Olivo, Source size and temporal coherence requirements of coded aperture type $x$-ray phase contrast imaging systems, Opt. Exp. 18 (2010) 19681.

[34] P. R. T. Munro, K. Ignatyev, R. D. Speller and A. Olivo, Phase and absorption retrieval using incoherent $x$-ray sources, PNAS 109 (2012) 13922.

[35] P. C. Diemoz et al., A simplified approach for computed tomography with an x-ray grating interferometer, Opt. Exp. 19 (2011) 1692.

[36] P. Zhu et al., Low-dose, simple, and fast grating-based $x$-ray phase-contrast imaging, PNAS 107 (2010) 13576. 